\title{
The Impact of Note-Taking on the Learning Process in Intelligent Tutoring System Tutomat
}

\author{
Ines Šarić-Grgicín ${ }^{*}$, Ani Grubišić², Branko Žitko ${ }^{3}$ \\ ${ }^{1-3}$ Faculty of Science, University of Split, Split, Croatia
}

\begin{abstract}
The research investigates how note-taking practice affects the learning process in Tutomat, an intelligent tutoring system. The complete analysis includes (i) the identification of learning analytics variables to describe student-Tutomat interaction; (ii) the description of experimental student groups using learning analytics variables; (iii) data-driven clustering and (iv) the comparison of the experimental groups and revealed clusters. The results show that there is a difference in how a student interacts with Tutomat based on note-taking practice. It is revealed that the note-taking practice can be detected using the proposed learning analytics variables with the prediction accuracy of the clustering approach of $85 \%$.
\end{abstract}

Keywords - Adaptive instructional systems, artificial intelligence in education, intelligent tutoring systems, learning analytics.

\section{INTRODUCTION}

Adaptive Instructional Systems (AISs) are recognised as a wider category of technology-enhanced learning systems that inherit all characteristics of the early Computer Assisted Instruction (CAI) systems and more recent Intelligent Tutoring Systems (ITSs). CAI systems proposed the use of computers as cognitive tools that enhance the cognitive powers of humans during thinking, problem-solving, and learning, as well as the platforms for constructivist learning [1]. On the other hand, ITS sought to mimic a human tutor who is a subject matter expert and who intelligently responds to students and their actions [2]. New hybrid systems integrate a variety of cognitive tools into tutoring systems by gathering interdisciplinary teams of computer scientists and educational psychologists to design systems that reflect best practices regarding effective pedagogy and adaptive learning for any domain knowledge.

The research field that significantly contributes to the improved capabilities of AIS is Learning Analytics (LA). As defined in 2011, the year of the First International Conference on Learning Analytics \& Knowledge, LA is the measurement, collection, analysis, and reporting of data about students and their contexts, for purposes of understanding and optimising learning and the environments in which it occurs. Each student's action during the tutoring process is saved into the system database. At the end of the tutoring process, various LA variables can be analysed, such as the number of domain knowledge components seen in the tutoring process, total time spent in different aspects of the tutoring process, total score gained online, the number of logins, etc. This valuable information about individual characteristics and group differences is consequentially analysed and used by AIS.

Regarding the general strategies that lead to improved learning, Trafton and Trickett [3] summarised several approaches, including self-explanation [4]; a cycle of prediction, testing, and reflection [5], [6]; reflective inquiry [7]; and the development of an appropriate and well-crafted "driving question" [8]. While the previous strategies have been associated with successful learning in different domains and instructional environments, Trafton and Trickett [3] discussed another effective strategy - note-taking practice. This strategy has been well studied in many traditional learning environments and it has frequently been found to boost student performance on recall of information [3], [9]-[12]. However, there are only a few studies that investigated the effectiveness and types of notes in digital environments [3], [13], [14]. The present research investigates how note-taking practice affects the learning process in the Adaptive Courseware based on Natural Language Processing Tutor (AC\&NL Tutor) environment (www.acnltutor.net), more specifically its intelligent tutoring system Tutomat. According to four of Bloom's knowledge dimensions [15], the AC\&NL Tutor environment is aimed at tutoring conceptual and factual knowledge represented in a form of text and concept graphs. The study complements the note-taking research by describing and comparing studentTutomat interaction using LA data. Since it has been revealed that the notes are especially helpful at the beginning of the tutoring process but should be phased out as students become more proficient, it is investigated if the note-taking practice can be detected using the proposed LA variables.

In the next section, the related work is provided. After that, the methodology is presented, including the description of the Tutomat. In the same section, the research questions, the experiment protocol, and the description of used methods are described. Afterward, the results and the discussion are presented, while the overall conclusion is provided at the end of the paper.

\footnotetext{
* Corresponding author’s email: ines.saric@pmfst.hr
} 


\section{RELATED WORK}

In traditional face-to-face lectures, there is an efficient strategy that improves learning - the note-taking practice. It has been revealed that this learning strategy is both favoured by students and encouraged by their instructors [16]. Instead of depending on their memory abilities, notes offer the advantage to students to "record, clarify, organise, and comprehend information highlighted during lectures" [17]. Moreover, the researchers revealed two aspects of note-taking, described as the quantity and quality characteristics. The aspect of quantity includes the frequency and duration of note-taking practice during learning, while the quality includes the depth of cognitive processing. In some cases, students merely copy verbatim what is read or heard, without significant cognitive engagement [18]. At other times, students use conceptual notetaking [19], summarisation [20], or self-questioning [11]. Only several studies investigated the use of note-taking practices in digital environments [3], [13], [14].

Trafton and Trickett [3] investigated note-taking practices during problem-solving tasks in a technology-enhanced learning environment. A total of 90 students participated in the research and they had to solve 5 scientific-reasoning tasks. The learning environment incorporated a notepad with a blank text box, on which participants could enter information or comments. The results of the research confirmed that notetaking practice was a helpful general strategy for learning since the students who used the notepad were more successful than those who did not. It appeared that the benefits of note-taking extended beyond boosting the simple recall of learned material and assisted students to make sense of the information they had learned and perhaps to enhance their problem-solving abilities [3]. Interestingly, it was also revealed that note-taking practice was more helpful during the early stage of learning, while students were still trying to understand the task and the steps necessary to solve it. Overall, the research indicated the positive aspect of using notes in the digital environment, as well as its contribution at the beginning of the tutoring process.

Apart from the problem-solving environment, the role of note-taking has been investigated in the narrative-centred game-based learning environment - Crystal Island [13]. The experiment included 116 students who studied microbiology and learned about pathogens, viruses, bacteria, fungi, and parasites. It was revealed that $53 \%$ of students took notes while using Crystal Island. There were 6 types of notes taken, including Narrative (facts from the unfolding storyline), Curricular (facts from the learning content), Hypothesis (a possible solution), Procedural (tasks to be completed), Garbage (meaningless information), and Other. The dominant notes belonged to the Curricular and Narrative categories, while the students taking Hypothesis notes showed better performance on post-test measures. The main contribution of the research includes different types of notes in the digital environment and their impact on overall learning.

Trevors, Duffy, and Azevedo [14] investigated the environment of MetaTutor, an intelligent tutoring system designed to scaffold cognitive and metacognitive self-regulated learning processes. The experiment included 60 students who used MetaTutor for 120 minutes and learned about the human circulatory system. It was revealed that the students with low prior knowledge took a greater quantity of notes compared to other students, but only in the absence of the MetaTutor selfregulated learning scaffolding. More importantly, it was revealed that most of the taken notes were a verbatim copy of instructional content, which was proven to negatively relate to the post-test measure of learning. This study confirmed the previous research on the contribution of notes at the beginning of the learning process but opened the research question about its effectiveness in the case when students copy instructional content.

It should be noted that the design and development of digital learning environments, such as intelligent tutoring systems, are extremely time and resource exhaustive, so the related work is taken into account regardless of the date of publication. Based on the related work, this study investigates how note-taking practice affects the learning process in the intelligent tutoring system called Tutomat. The proposed LA variables are used to describe and compare the learning process in Tutomat, while it is also investigated if the note-taking practice can be detected from the student-Tutomat interaction.

\section{METHODOLOGY}

In this section, the Tutomat and its basic characteristics are described. Then, the research questions and experiment protocol are described. The methods used to address each research question are described in the last section.

\section{A. Tutomat}

The AC\&NL Tutor environment comprises two parts: (i) the Semi-Automatic Authoring Tool (SAAT) that performs knowledge extraction utilising natural language processing, and (ii) the Tutomat, the intelligent tutoring system that enables adaptive courseware and communication in the controlled natural language.

The Tutomat can be used in any domain since the only prerequisite to enable tutoring is to prepare a text using the SAAT. When a teacher has approved the SAAT output, courseware elements are automatically generated and ready for utilisation in the Tutomat. The courseware elements include the sentences and questions of different levels of complexity, along with their visual representations in the form of concept graphs.

There are four stereotypes used in the Tutomat (beginner, intermediate, advanced, and expert) and each stereotype has its own set of rules for selection, sequencing, and presentation of courseware elements, where higher stereotypes learn from bigger and more complex courseware elements.

Also, the tutoring process in the Tutomat incorporates three consequent processes that form a unique tutoring cycle. While learning in Tutomat, a student goes through several tutoring cycles. Each cycle consists of three phases: 1) the learning and teaching phase that is adapted to the current student's knowledge level (Fig. 1); 2) the testing phase (Fig. 2), and 3) the evaluation phase when the student model is updated according to the test results (Fig. 3). After the testing phase, each student's answer to a specific question is mapped to one 
or more propositions, and depending on the stereotype, the student can get from 1 to 4 points for each correct proposition. At the end of each tutoring cycle, the student model (current stereotype) is updated according to the maximum number of learned concepts for each stereotype and overlaid with the expert knowledge. The tutoring process is finished when complete courseware elements are learned at the highest knowledge level.

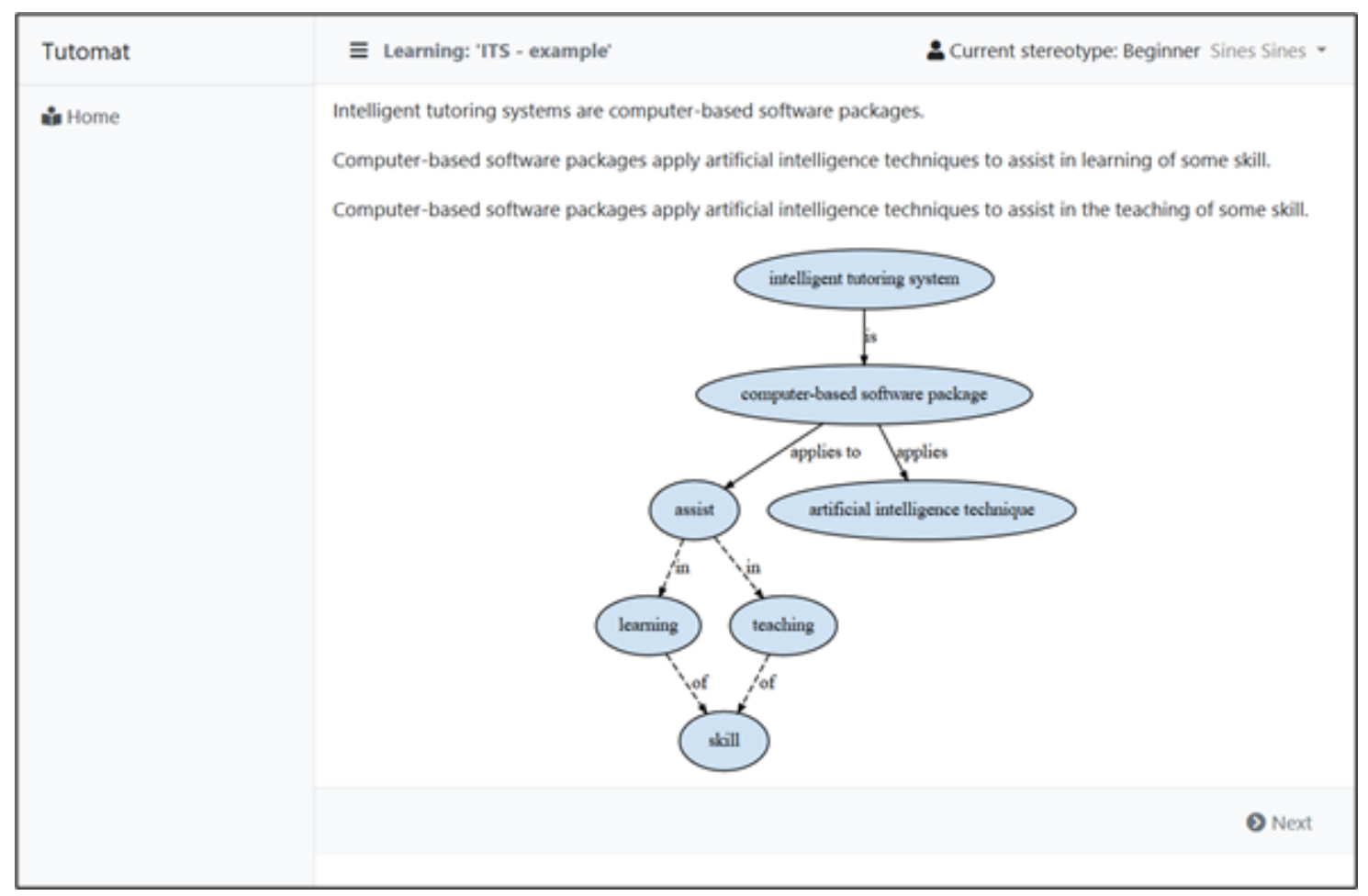

Fig. 1. The learning and teaching phase in the Tutomat.

\begin{tabular}{|l|l|}
\hline Tutomat & E Testing: 'ITS - example' \\
\hline \multirow{2}{*}{ Home } & What are intelligent tutoring systems? \\
\hline Computer-based software packages & 2 Current stereotype: Beginner Sines Sines - \\
\hline What do computer-based software packages apply? & \\
\hline
\end{tabular}

Fig. 2. The testing phase in the Tutomat.

\begin{tabular}{|l|l|}
\hline Tutomat & E Test results: 'ITs - example' \\
\hline What are intelligent tutoring systems? & 2 Next stereotype: Beginner Sines Sines - \\
Correct answer: Computer-based software packages \\
- Computer-based software packages \\
What do computer-based software packages apply? \\
Correct answer: Artificial intelligence techniques to assist in the teaching of some skill, Artificial intelligence \\
techniques to assist in learning of some skill. \\
-
\end{tabular}

Fig. 3. The evaluation phase in the Tutomat. 


\section{B. Research Questions and Experiment Protocol}

In this study, we are interested in answering the following questions.

1. Which LA variables can be derived from the database and used to describe a student interaction with the Tutomat?

2. Based on LA variables, what are the characteristics that distinguish two groups of students with different note-taking practices?

3. What are the characteristics that distinguish students belonging to two data-driven clusters based on student interaction with the Tutomat?

4. How do previously identified student clusters relate to the two groups of students with different note-taking practices?

The study was conducted at the Faculty of Science, the University of Split, and involved 33 graduate students who enrolled in the "E-learning Systems" course. The domain knowledge used in the study included basic information about intelligent tutoring systems. Since the previous research determined that around half of the students preferred notetaking practice, the students were randomly assigned to two groups that used the Tutomat during regular class that lasted for 90 minutes. The first group used the Tutomat solely (Group 1), while the second group used notes (paper-based or digital) during the tutoring process (Group 2).

\section{Methods}

During the complete and individualised tutoring process, the student interaction with the Tutomat is continuously saved in the Tutomat database. Regarding the first research question, the $\log$ of student interaction in the Tutomat is extracted from the database using SQL, while the further analysis, including LA variables, is computed using the Python language.

Regarding the second research question, two groups of students with different note-taking practices are described using the previously introduced LA variables. The statistical methods are used to compare LA variables between two student groups. Since nonparametric statistical methods do not assume any specific data distribution, the Mann-Whitney $U$ test is used to determine the statistical significance of the differences between two student groups.

To investigate data-driven student clusters based on LA variables, since there are two experimental student groups, the number of clusters is predefined as two. Firstly, data are preprocessed using $z$-score normalization. Then, due to its wide use and high interpretability, the $K$-means clustering algorithm is used to find two student clusters. The arbitrary and intuitive approach to analysing the average values of each variable is used to describe revealed clusters. Additionally, the Mann-
Whitney $U$ tests are used to determine the statistical significance of the difference between two student clusters.

Since the way of a student interacting with the Tutomat is known, the relations between actual student groups and computed clusters are described using a confusion matrix.

\section{RESULTS AND DISCUSSION}

In terms of the LA variables that can be used to describe the tutoring process in the Tutomat, the variables related to gained knowledge are not relevant because all students who participated in the experiment finished the tutoring process at the highest level - the expert stereotype. The potential LA variables that describe the tutoring process in the Tutomat are related to the number of tutoring cycles and different aspects of time spent online.

The LA variables collected by the Tutomat and selected for further analysis include: 1) the number of tutoring cycles needed to finish the tutoring process - \#Tutoring cycles; 2) the total time spent in the learning and teaching phase of the tutoring process (in minutes) - \#Time learning; 3) the total time spent in the testing phase of the tutoring process (in minutes) \#Time testing; and 4) the total time spent in the evaluation phase of the tutoring process (in minutes) - \#Time evaluation. All LA variables are calculated using online learning data logs from the Tutomat database. The average and standard deviation (SD) values of LA variables for the dataset are shown in Table I.

Since nonparametric statistical methods do not assume any specific data distribution, we use the Mann-Whitney $U$ test to check the differences between each pair of LA variables of two student groups. The results revealed that there was a statistically significant difference in each LA variable between Group 1 and Group 2 (Table II). Also, we investigated the difference in the total amount of time spent in the Tutomat between Group 1 and Group 2, and the results showed that there was no statistically significant difference in total time between Group 1 and Group $2(p$-value $=0.06)$.

Next, we investigated the characteristics that distinguished students belonging to two data-driven student clusters based on LA variables. Since the number of tutoring cycles and the different aspects of time spent online are measures of different units, data were firstly standardized. Having in mind differences between two student groups, the LA variables were used to identify two student clusters. The $K$-means clustering and the Euclidean distance as the distance metric were used in this analysis. The average values of each LA variable for the revealed clusters are shown in Table III. 
TABLE I

LA VARIABLES - GROUP 1, GROUP 2, GROUPS 1 AND 2

\begin{tabular}{|c|c|c|c|c|}
\hline & \#Tutoring cycles & \#Time learning & \#Time testing & \#Time evaluation \\
\hline \multicolumn{5}{|c|}{ Group 1} \\
\hline Average & 6.68 & 14.87 & 21.46 & 6.52 \\
\hline Standard Deviation & 3.65 & 5.30 & 5.38 & 3.05 \\
\hline \multicolumn{5}{|c|}{ Group 2} \\
\hline Average & 4.47 & 5.61 & 29.65 & 3.42 \\
\hline Standard Deviation & 1.09 & 2.16 & 8.02 & 3.17 \\
\hline \multicolumn{5}{|c|}{ Groups 1 and 2} \\
\hline Average & 5.55 & 10.10 & 25.68 & 4.93 \\
\hline Standard Deviation & 2.93 & 6.21 & 8.12 & 3.53 \\
\hline
\end{tabular}

TABLE II

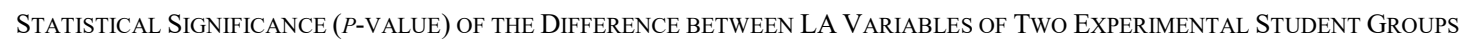

\begin{tabular}{|l|l|c|c|c|c|}
\hline & & \#Tutoring cycles & \#Time learning & \#Time testing & \#Time evaluation \\
\hline Group 1 & Group 2 & $p$-value $=0.01$ & $p$-value $=0.01$ & $p$-value $=0.01$ & $p$-value $=0.01$ \\
\hline
\end{tabular}

TABLE III

Statistical Measures of LA Variables for Two ReVealed Student Clusters

\begin{tabular}{|l|c|c|c|c|c|c|c|c|}
\hline & \multicolumn{2}{|c|}{ \#Tutoring cycles } & \multicolumn{2}{c|}{ \#Time learning } & \multicolumn{2}{c|}{ \#Time testing } & \multicolumn{2}{c|}{ \#Time evaluation } \\
\hline & Average & SD & Average & SD & Average & SD & Average & SD \\
\hline Cluster 1 & 6.73 & 3.76 & 14.35 & 6.31 & 18.91 & 4.70 & 7.51 & 3.31 \\
\hline Cluster 2 & 4.55 & 1.11 & 6.55 & 2.78 & 31.32 & 5.36 & 2.76 \\
\hline
\end{tabular}

TABLE IV

Statistical Significance ( $P$-VALUE) OF THE DifFERENCE BETWEen Two REVEALED STUdENT Clusters

\begin{tabular}{|l|l|c|c|c|c|}
\hline & & \#Tutoring cycles & \#Time learning & \#Time testing & \#Time evaluation \\
\hline Cluster 1 & Cluster 2 & $p$-value $=0.02$ & $p$-value $=0.01$ & $p$-value $=0.01$ & $p$-value $=0.01$ \\
\hline
\end{tabular}

TABLE V

CONFUSION MATRIX FOR THE EXAMINED DATASET

\begin{tabular}{|l|c|c|}
\hline & Cluster 1 & Cluster 2 \\
\hline Group 1 & 13 & 3 \\
\hline Group 2 & 2 & 15 \\
\hline
\end{tabular}

We also wanted to determine if clusters were statistically different in terms of each LA variable. Therefore, we used the Mann-Whitney $U$ test to check the difference. The results revealed that there was a statistically significant difference in each LA variable between Cluster 1 and Cluster 2 (Table IV).

To address the fourth research question, the relation between the actual student group and the revealed student cluster was analysed. The confusion matrix for the complete dataset is shown in Table V.

In total, the $K$-means algorithm similarly clustered 28 students $(85 \%)$ that belonged to the same experimental student group regarding note-taking practices, while 5 students $(15 \%)$ were incorrectly classified.

As the results showed, the identified LA variables were useful in describing the tutoring process in the Tutomat. Moreover, these variables can be used to detect note-taking while learning in the Tutomat. In case when a student does not use notes, for the included domain knowledge, a student will on average finish the tutoring process in 6.73 cycles $(\mathrm{SD}=3.76)$, spend in the learning and teaching phase 14.35 minutes $(\mathrm{SD}=$ $6.31)$, in the testing phase 18.91 minutes $(\mathrm{SD}=4.7)$, and in the evaluation phase 7.51 minutes $(\mathrm{SD}=3.31)$. A student with notetaking practices will on average finish the tutoring process in 4.55 cycles $(\mathrm{SD}=1.11)$, spend in the learning and teaching phase 6.55 minutes $(\mathrm{SD}=2.78$ ), in the testing phase 31.32 minutes $(\mathrm{SD}=5.36)$, and spend in the evaluation phase 2.76 minutes $(\mathrm{SD}=1.67)$. The student group that used the Tutomat solely got through more tutoring cycles than the group that used notes. This group spent more time in the learning and teaching phase, as well as in the evaluation phase. On the other hand, the note-taking group spent more time in the testing phase in the Tutomat. 


\section{CONCLUSION}

In this study, the note-taking practice in the Tutomat has been investigated, including 1) the identification of LA variables, 2) the description of experimental student groups using LA variables, 3) data-driven clustering using LA variables, and 4) the comparison of the experimental student groups and revealed student clusters. In the case of domain knowledge that included basic information about intelligent tutoring systems, students spent on average 41 minutes in the Tutomat to learn the prepared material. The results revealed that whether or not a student took notes while learning in the Tutomat, the total amount of time spent in the tutoring process remained the same. However, in the case of taking notes, a student spent significantly more time in the testing phase in the Tutomat. In contrast, a student that used the Tutomat solely spent more time in the learning and teaching, as well as the evaluation phase.

Since the results showed that the revealed clusters differed in all examined variables, the proposed LA variables could successfully be used to detect students who used notes while learning in the Tutomat. The accuracy of $85 \%$ of the predicted cluster on a complete dataset should be additionally checked with a larger dataset.

Also, since the Tutomat is used to tutor conceptual and factual knowledge, the depth of student notes is probably shallow, having notes as just a copy of material presented in the learning and teaching phase. Since the background research has shown that this type of notes negatively relates to the post-test measure of learning, it would be important to additionally check the gained knowledge of two experimental student groups and investigate the types of used notes. In case of similar performance of two student groups, it should be advised to use notes, since these students probably feel more relaxed while using the Tutomat. Otherwise, the data-driven clustering approach proposed in the research could serve to distinguish students that recall information from the memory from the students that copy the answers from their notes.

Based on the background research of the problem-solving tasks, the note-taking practice can also be used as a scaffolding strategy helpful at the beginning of the tutoring process, or the lowest level of the tutoring process, but phased out as students become more proficient. However, to successfully test the knowledge at the highest level, it is expected that a student has memorised all the concepts and answers questions by recalling information from the memory. At these higher levels of the tutoring process, the data-driven clustering approach as described in the research can be used to detect students who take notes while learning in the Tutomat.

\section{ACKNOWLEDGMENT}

The research has been supported by the United States Office of Naval Research under grants N00014-15-1-2789 “Adaptive Courseware \& Natural Language Tutor" and N00014-20-12066 "Enhancing Adaptive Courseware based on Natural Language Processing".

\section{REFERENCES}

[1] J. A. DeFalco and A. M. Sinatra, "Adaptive Instructional Systems: The Evolution of Hybrid Cognitive Tools and Tutoring Systems," in Adaptive Instructional Systems, R. Sottilare, J. Schwarz J., Eds. Springer, Cham, 2019, pp. 52-61. https://doi.org/10.1007/978-3-030-22341-0 5

[2] R. Sottilare, "A Comprehensive Review of Design Goals and Emerging Solutions for Adaptive Instructional Systems," Tech. Inst. Cognition and Learning, vol. 11, no. 1, pp. 5-38, 2018.

[3] J. G. Trafton and S. B. Trickett, "Note-Taking for Self-Explanation and Problem Solving," Human-Computer Interaction, vol. 16, no. 1, pp. 138, Mar. 2001. https://doi.org/10.1207/S15327051HCI1601 1

[4] M. T. H. Chi, M. Bassok, M. W. Lewis, P. Reimann, and R. Glaser, "SelfExplanations: How Students Study and Use Examples in Learning to Solve Problems," Cognitive Science, vol. 13, no. 2, pp. 145-182, Apr. 1989. https://doi.org/10.1207/s15516709cog1302_1

[5] M. C. Salzman, C. Dede, and R. B. Loftin, "VR's Frames of Reference: A Visualization Technique for Mastering Abstract Multidimensional Information," in Proceedings of the SIGCHI conference on Human Factors in Computing Systems, Pittsburgh, Pennsylvania, USA, May 1999, pp. 489-495. https://doi.org/10.1145/302979.303141

[6] B. Y. White, "ThinkerTools: Causal Models, Conceptual Change, and Science Education," Cognition and Instruction, vol. 10, no. 1, pp. 1-100, Mar. 1993. https://doi.org/10.1207/s1532690xci1001_1

[7] I. Tabak, W. A. Sandoval, B. K. Smith, F. Steinmuller, and B. J. Reiser, "Reflection as a Vehicle toward Local and Global Understanding," Jan. 1998.

[8] B. Barron et al., "Doing with Understanding: Lessons From Research on Problem- and Project-Based Learning," Journal of the Learning Sciences, vol. 7, no. 3-4, pp. 271-311, Jul. 1998. https://doi.org/10.1080/10508406.1998.9672056

[9] K. A. Kiewra, "A Review of Note-Taking: The Encoding-Storage Paradigm and Beyond," Educational Psychology Review, vol. 1, no. 2, pp. 147-172, Jun. 1989. https://doi.org/10.1007/BF01326640

[10] S. D. Rinehart and K. F. Thomas, "Summarization Ability and Text Recall by Novice Studiers," Reading Research and Instruction, vol. 32, no. 4, pp. 24-32, Jun. 1993. https://doi.org/10.1080/19388079309558130

[11] H. A. Spires, "Learning from a Lecture: Effects of Comprehension Monitoring," Reading Research and Instruction, vol. 32, no. 2, pp. 1930, Dec. 1992. https://doi.org/10.1080/19388079309558113

[12] P. Van Meter, L. Yokoi, and M. Pressley, "College Students' Theory of Note-Taking Derived from Their Perceptions of Note-Taking," Journal of Educational Psychology, vol. 86, no. 3, pp. 323-338, Sep. 1994. https://doi.org/10.1037/0022-0663.86.3.323

[13] S. W. McQuiggan, J. Goth, E. Ha, J. P. Rowe, and J. C. Lester, "Student Note-Taking in Narrative-Centered Learning Environments: Individual Differences and Learning Effects," in Intelligent Tutoring Systems, Berlin, Heidelberg, 2008, pp. 510-519. https://doi.org/10.1007/978-3540-69132-7 54

[14] G. Trevors, $\bar{M}$. Duffy, and R. Azevedo, "Note-Taking within MetaTutor: Interactions between an Intelligent Tutoring System and Prior Knowledge on Note-Taking and Learning," Educational Technology Research and Development, vol. 62, no. 5, pp. 507-528, Oct. 2014. https://doi.org/10.1007/s11423-014-9343-8

[15] L. W. Anderson et al., A Taxonomy for Learning, Teaching, and Assessing: A Revision of Bloom's Taxonomy of Educational Objectives, Abridged Edition, 1st edition. New York: Pearson, 2000.

[16] I. R. Weiss, "A National Convocation on Professional Development for Mathematics and Science Teachers, K-12," 2002.

[17] J. M. Bonner and W. G. Holliday, "How College Science Students Engage in Note-Taking Strategies," Journal of Research in Science Teaching, vol. 43, no. 8, pp. 786-818, Oct. 2006. https://doi.org/10.1002/tea.20115

[18] E. N. Laidlaw, R. L. Skok, and T. F. McLaughlin, "The Effects of Notetaking and Self-Questioning on Quiz Performance," Science Education, vol. 77, no. 1, pp. 75-82, Jan. 1993. https://doi.org/10.1002/sce.3730770105

[19] J. P. Rickards and C. B. McCormick, "Effect of Interspersed Conceptual Prequestions on Note-Taking in Listening Comprehension," Journal of Educational Psychology, vol. 80, no. 4, pp. 592-94, 1988. https://doi.org/10.1037/0022-0663.80.4.592

[20] A. King, "Comparison of Self-Questioning, Summarizing, and Notetaking-Review as Strategies for Learning From Lectures," American Educational Research Journal, vol. 29, no. 2, pp. 303-323, Jun. 1992. https://doi.org/10.3102/00028312029002303 


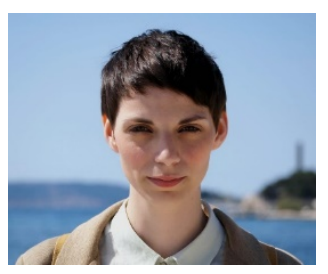

Ines Šarić-Grgić is a Research Assistant at the Faculty of Science, University of Split, Croatia, and $\mathrm{ahD}$ student of Computer Science at the Faculty of Electrical Engineering, Mechanical Engineering, and Naval Architecture, University of Split, Croatia, where she earned her MSc in 2008. In 2012, she received an MSc specialization in Business Economics at the Faculty of Economics, University of Split, Croatia. Since 2015, she has worked on two projects funded by the Office of Naval Research, USA. Her research interest includes artificial intelligence in education and intelligent tutoring systems.

E-mail: ines.saric@pmfst.hr

ORCID iD: https://orcid.org/0000-0002-9247-8890

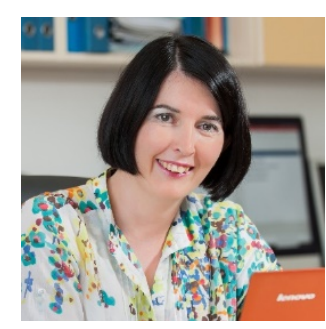

Ani Grubišić is an Associate Professor at the University of Split, Faculty of Science. She graduated at the same Faculty in 2001, got her MS in 2007 and $\mathrm{PhD}$ in 2012 at the University of Zagreb, Faculty of Electrical Engineering and Computing. Areas of scientific interest are intelligent tutoring systems, adaptive courseware and learning analytics in e-learning systems. She is a Principal Investigator of two projects "Adaptive Courseware based on Natural Language Processing (AC \& NL Tutor)", funded by the Office of Naval Research, USA. She is an author and co-author of more than thirty scientific papers.

E-mail: ani.grubisic@pmfst.hr

ORCID iD: https://orcid.org/0000-0003-4313-7851

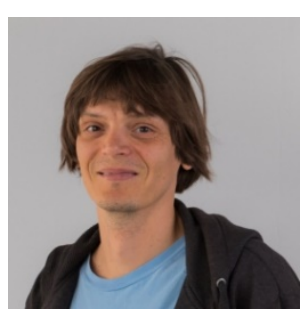

Branko Žitko, $\mathrm{PhD}$, is an assistant professor at the Faculty of Science at University of Split. His teaching responsibilities are Problem Solving, Software Engineering and Natural Language Processing. Branko Žitko's expertise relates to Intelligent Tutoring Systems, Knowledge Representation and Natural Language Processing. $\mathrm{He}$ is currently working on a Knowledge Extraction from Natural Language Text.

ORCID iD: https://orcid.org/0000-0001-8946-0916 